\title{
Relationship between Urate Crystal Deposits Detected by Dual-energy Computed Tomography and Bone Erosions in Symptomatic Gout Patients without Clinically Apparent Tophi
}

\author{
Min Kyung Chung ${ }^{1}$, In Je Kim ${ }^{1}$, Hyeran Hyun ${ }^{2}$, Ji Young Hwang ${ }^{2}$, Jisoo Lee \\ ${ }^{1}$ Division of Rheumatology, Department of Internal Medicine, and ${ }^{2}$ Department of Radiology, Ewha Womans University School of Medicine, \\ Seoul, Korea
}

\begin{abstract}
Objective. Dual-energy computed tomography (DECT) allows sensitive detection of monosodium urate (MSU) crystal deposits in gout. However, the role of MSU deposits on DECT during the disease process of gout is not clear. The aim of our study was to evaluate the relationship between joint damage and MSU deposits detected by DECT in symptomatic non-tophaceous gout. Methods. DECT scans of 51 gout patients without clinically apparent tophi were assessed. Individual ankle and foot joints and Achilles tendon insertion sites were evaluated for the presence of MSU deposits and bone erosions. The total volume of MSU crystal on DECT was quantified using an automated software program. Clinical and laboratory data at the time of the DECT evaluation were obtained from medical record. Results. MSU deposits were detected in $92.2 \%$ of the patients evaluated. Median number and total volume of MSU deposit per patient was 5.0 and $0.6 \mathrm{~cm}^{3}$, respectively. Bone erosion was found in $54.9 \%$ of patients. MSU deposits in the first (1st) metatarsophalangeal (MTP) joints were significantly associated with presence of bone erosions (odds ratio [OR] 3.77, 95\% confidence interval $[\mathrm{Cl}] 1.06 \sim 13.38, \mathrm{p}=0.040$ ). Older age and frequent gout attack were associated with development of bone erosion in patients with MSU deposits (OR 1.12 and 2.57, 95\% CI $1.04 \sim 1.22$ and $1.02 \sim$ 6.50, p-value 0.004 and 0.047, respectively). Conclusion. MSU deposits and erosions were frequently detected by DECT in symptomatic non-tophaceous gout patients, and MSU deposits in 1st MTP joints were associated with presence of bone erosions especially in patients with older age and frequent gout attack. (J Rheum Dis 2019;26:124-130)
\end{abstract}

Key Words. Gout, Monosodium urate, Erosion, Dual-energy computed tomography

\section{INTRODUCTION}

Gout is a common inflammatory arthritis caused by deposition of monosodium urate (MSU) crystals in soft tissues and joints [1]. After many acute mono- or oligo-articular attacks, a proportion of patients may progress into chronic synovitis with joint destructions. With development of the imaging modalities such as magnetic resonance imaging (MRI) [2], computed tomography (CT) [3], and ultrasonography (US) [4,5], we are cur- rently able to visualize MSU crystals without joint aspiration. Dual-energy computed tomography (DECT), which can differentiate chemical components of material using material-specific differences in attenuation on different peak voltages, is a highly sensitive method for detecting MSU crystal deposits [6-9]. Because of several advantages of DECT over MRI or US by providing simultaneous evaluation of MSU deposits and bone erosions in multiple joints within short evaluation time, DECT is emerging as a promising tool for the diagnosis and treat-

Received : October 26, 2018, Revised : January 3, 2019, Accepted : January 22, 2019

Corresponding to : Jisoo Lee (iDhttp://orcid.org/0000-0001-6279-7025

Division of Rheumatology, Department of Internal Medicine, Ewha Womans University School of Medicine, 1071 Anyangcheon-ro, Yangcheon-gu, Seoul 07985, Korea. E-mail : leejisoo@ewha.ac.kr

Ji Young Hwang (iD http://orcid.org/0000-0001-5848-3429

Department of Radiology, Ewha Womans University School of Medicine, 1071 Anyangcheon-ro, Yangcheon-gu, Seoul 07985, Korea. E-mail : mshjy@ewha.ac.kr 
ment of gout $[3,6,10]$.

In studies utilizing DECT in gout, MSU deposits are detected in high frequency ranging from $47 \% \sim 100 \%$ in patients evaluated [11-13]. However, aside from diagnostic value of MSU deposits detected on DECT, their role during the disease process is not clear. It was shown that total volume of urate deposition on DECT was significantly lower in patients with asymptomatic hyperuricemia compared with the symptomatic gout, suggesting that a threshold volume of urate is requirement for developing clinically significant disease [9]. In patients with chronic tophaceous gout, bone erosions were shown to be present in joints with tophi suggesting a role of MSU deposits on DECT in structural joint damage $[14,15]$. Both asymptomatic hyperuricemia and chronic tophaceous gout represent extreme ends of the disease process. However, clinical significance of MSU deposits on DECT in majority of symptomatic gout patients without apparent clinical tophi has not been studied in detail previously. The aim of this study was to examine relationship between structural joint damage and MSU deposits detected on DECT in symptomatic gout patients without clinically apparent tophi.

\section{MATERIALS AND METHODS}

\section{Patients}

We retrospectively analyzed medical records of 51 patients who consecutively underwent DECT scans for evaluation of gout during the rheumatology outpatient visit or through consultation service during admission between January 1, 2014 and January 31, 2016 at Ewha Womans University Mokdong Hospital. All patients fulfilled 1977 American College of Rheumatology classification criteria for gout [16]. Patients without clinically apparent tophi on physical examination documented by medical records, and those who have never been on urate lowering therapy before the DECT evaluation were included in this study. This study was approved by the local Institutional Review Board (IRB no. 2018-01-031).

\section{Clinical and laboratory assessment}

Demographics, clinical and laboratory data were retrospectively obtained by medical chart review using a standardized format at the time of the DECT evaluation. Clinical data included age, sex, daily dose of alcohol intake, a duration of disease, frequency of gout attack per year, symptomatic joints, and comorbidities including diabetes mellitus, hypertension, hyperlipidemia, previous cardiovascular disease, and chronic kidney disease. Laboratory data included serum urate, creatinine, glucose levels. Hyperlipidemia was defined as the presence of one or more altered values of the lipid profile according to the cut-off values of laboratory recommendation: total cholesterol $>200 \mathrm{mg} / \mathrm{dL}$; triglyceride $>200 \mathrm{mg} / \mathrm{dL}$; low density lipoprotein cholesterol $>130 \mathrm{mg} / \mathrm{dL}$, and patients who were on lipid lowering agents was considered to have hyperlipidemia. The estimated glomerular filtration rate was calculated using the Modification of Diet in Renal Disease Study equation, and chronic kidney disease was defined as higher than stage 3 of estimated glomerular filtration rate below $60 \mathrm{~mL} / \mathrm{min} / 1.73 \mathrm{~m}^{2}$ for 3 months or more [17].

\section{Imaging - DECT scans}

DECT scans of both feet and ankles were performed on a dual X-ray tube 128-detector-row scanner (Somatom Definition Flash; Siemens Medical Solutions, Erlangen, Germany). Patients were positioned supine with their feet in a plantar flexion position. All scans were obtained from ankle to tip of a big toe with standard protocol at $64 \times 0.6 \mathrm{~mm}$ and pitch of 0.7 . X-ray tube 1 and 2 were operated at $80 \mathrm{kV} / 165 \mathrm{mAs}$ and $140 \mathrm{kV} / 85 \mathrm{mAs}$, respectively. The images were reconstructed on a bone algorithm to $0.75 \mathrm{~mm}$ slices with a $0.5 \mathrm{~mm}$ increment. Volume of the MSU crystal deposits was assessed using a commercial software program ('Gout', Syngo CT workplace; Siemens Medical System, Erlangen, Germany) after drawing volume of interest. Total volume included $9,000 \mathrm{~cm}^{3}$ volume for each foot. Two independent musculoskeletal radiologists with 19 years and 5 years of experience who were blinded to the clinical features reviewed axial as well as coronal and sagittal planes of DECT images of 51 patients. The presence of MSU crystal deposits and erosions in both metatarsophalangeal (MTP) joints (510 joints), Lisfranc joints (102 joints), transverse tarsal joints (102 joints), and ankle joints (102 joints) and MSU crystals at Achilles tendons (102) were scored. MSU crystal deposit was defined as round and nodular deposits in and near joints and insertion site of Achilles tendon detected as green color on DECT. Artifacts in nail bed and skin, submillimeter lesion, and motion artifacts were excluded when measuring the volume of MSU deposits in each joint, but could not be excluded when measuring the total volume due to limitations of the software used in the analysis [18]. Erosion was defined as cortical disruption 
in any two planes of the axial, coronal, and sagittal planes. The number of MSU crystal deposits and erosions were counted as one when they were present within the same joint. Disagreement between readers for the presence of MSU deposits and erosions was resolved by consensus. Inter-reader agreement for detection of MSU deposits and erosion were $86.2 \%$ and $90.5 \%$, and Cohen's kappa were 0.6 and 0.5 respectively $(\mathrm{p}<0.05)$.

\section{Statistical analysis}

Continuous variables were expressed as mean with standard deviation (SD) or median with range, and categorical data were expressed as number and percentages. For the site-by-site analysis, each joint was considered as independent unit and a generalized estimating equations (GEE) approach was used to adjust the agreement between adjacent bones within each individual since joints were nested within patients. To identify factors affecting presence of bone erosion, univariate analysis was performed. Statistically significant factors with $\mathrm{p}$-value of $<0.1$ were included in multivariate logistic regression model using backward likelihood ratio process. All data were analyzed using IBM SPSS (version 21; IBM Co., Armonk, NY, USA). A p-value of $<0.05$ was considered to indicate statistical significance.

Table 1. Characteristics of patients $(n=51)$

\begin{tabular}{lc}
\hline \multicolumn{1}{c}{ Variable } & Value \\
\hline Age $(y r)$ & $48.4 \pm 15.0$ \\
Male & $47(92.2)$ \\
Comorbidity & $47(92.2)$ \\
Diabetes mellitus & $5(9.8)$ \\
Hypertension & $26(51.0)$ \\
Hyperlipidemia & $43(84.3)$ \\
Previous CV event & $8(15.7)$ \\
Chronic kidney disease & $8(15.7)$ \\
Duration of gout (yr) & $2.0(0.0 \sim 20.0)$ \\
Gout attack/year & $2.0(0.6 \sim 12.0)$ \\
Alcohol intake $>20$ g/d & $25(50.0)$ \\
Serum urate $(\mathrm{mg} / \mathrm{dL})$ & $8.10(3.5 \sim 15.4)$ \\
Symptomatic joints & \\
MTP & $36(70.6)$ \\
Ankle & $16(31.4)$ \\
Mid-foot & $8(15.7)$ \\
Knee & $2(3.9)$
\end{tabular}

Values are presented as mean \pm standard deviation, number (\%), or median (range). CV: cardiovascular, MTP: metatarsophalangeal.

\section{RESULTS}

\section{Patient characteristics}

Most patients were male (92.2\%) with a mean \pm SD age of $48.4 \pm 15.0$. The median duration of gout was 2 years ranging from 0 to 20 years. Over ninety percent of patients had comorbidities including diabetes mellitus, hypertension, hyperlipidemia, cardiovascular event, and chronic kidney disease with hypertension to be the most frequently observed condition. On average, patients experienced gout attack 2 times a year. Fifty percent of the patients were heavy alcohol consumer, drinking over $20 \mathrm{~g}$ of alcohol per day. Median (range) serum urate level was $8.10(3.5 \sim 15.4) \mathrm{mg} / \mathrm{dL}$. The MTP joints were the most commonly affected joints followed by ankle, mid-foot, and knee joints (Table 1).

\section{Characteristics of DECT findings}

Table 2 represents characteristics of DECT findings in patients evaluated. MSU deposits were detected by DECT in 47 patients (92.2\%). Median (range) number of MSU deposits per patient was $5.0(0 \sim 17)$, and the median total volume of MSU crystal deposits was $0.6(0.05 \sim 4.86)$ $\mathrm{cm}^{3}$. MSU crystal deposits were found most frequently on

Table 2. Characteristics of DECT findings $(n=51)$

\begin{tabular}{ll}
\hline \multicolumn{1}{c}{ Variable } & \multicolumn{1}{c}{ Value } \\
\hline Presence of MSU deposit & $47(92.2)$ \\
Number of MSU deposit /patient & $5.0(0 \sim 17)$ \\
Total volume of MSU deposit /patients $\left(\mathrm{cm}^{3}\right)$ & $0.6(0.05 \sim 4.86)$ \\
Sites of MSU deposit & $43(84.3)$ \\
MTP joints & $33(64.7)$ \\
1st MTP & $33(64.7)$ \\
Ankle joints & $15(29.4)$ \\
Lisfranc joints & $14(27.5)$ \\
Transverse tarsal joints* & $24(47.1)$ \\
Achilles tendons & $28(54.9)$ \\
Presence of erosion & $1.0(0 \sim 6)$ \\
Mean number of erosion/patient & $17(33.3)$ \\
Sites of erosion & $17(33.3)$ \\
MTP joints & $12(23.5)$ \\
1st MTP & $11(21.6)$ \\
Ankle joints & $7(13.7)$ \\
Lisfranc joints & \\
Transverse tarsal joints*
\end{tabular}

Values are presented as number (\%) or median (range). DECT: dual-energy computed tomography, MSU: monosodium urate, MTP: metatarsophalangeal. *Intertarsal, talo-navicular, calcaneo-cuboidal. 
MTP joints (84.3\%), especially on 1st (first) MTP joints $(64.7 \%)$, followed by ankle $(64.7 \%)$, Achilles tendons (47.1\%), Lisfranc joints $(29.4 \%)$, and transverse tarsal joints $(27.5 \%)$. Bone erosions were found in 28 (54.9\%) patients, and the median (range) number of erosions per patient was $1.0(0 \sim 6)$. Erosion was also most frequently detected at 1st MTP joints, followed by ankle, Lisfranc, and transverse tarsal joints in the same order of MSU deposit frequency. DECT images of MSU deposits and erosions on first MTP joints are shown in Figure 1.

\section{Relationship between subclinical MSU deposits and bone erosion on DECT}

To determine the relationship between MSU deposits and bone erosions on DECT, presence of erosions on individual joints with and without MSU depositions was compared (Table 3). MSU crystal deposit was detected in $297 / 816$ (36.4\%) joints, and erosion was found in 67/816 (8.2\%) joints. In MTP, ankle, and transverse tarsal joints, joints with MSU deposits had more erosions compared with those without (MTP $9.0 \%$ vs. $1.9 \%$, ankle $14.3 \%$ vs. $13.0 \%$, transverse tarsal $25.0 \%$ vs. $9.8 \%$ ). However, in GEE analysis, only 1st MTP joints with MSU deposits had significantly higher risk of erosion compared with those without (odds ratio [OR] 3.77, 95\% confidence interval [CI] $1.06 \sim 13.38, \mathrm{p}=0.040$ ).

Factors affecting development of bone erosion in patients with MSU deposits were analyzed by univariate and multivariate analysis (Table 4). Univariate analysis showed that older age (OR 1.10, 95\% CI 1.03 1.17, $\mathrm{p}=0.003$ ), accompanied hypertension (OR 5.04, 95\% CI $1.40 \sim 18.14, \mathrm{p}=0.013$ ), and absence of MSU deposit on
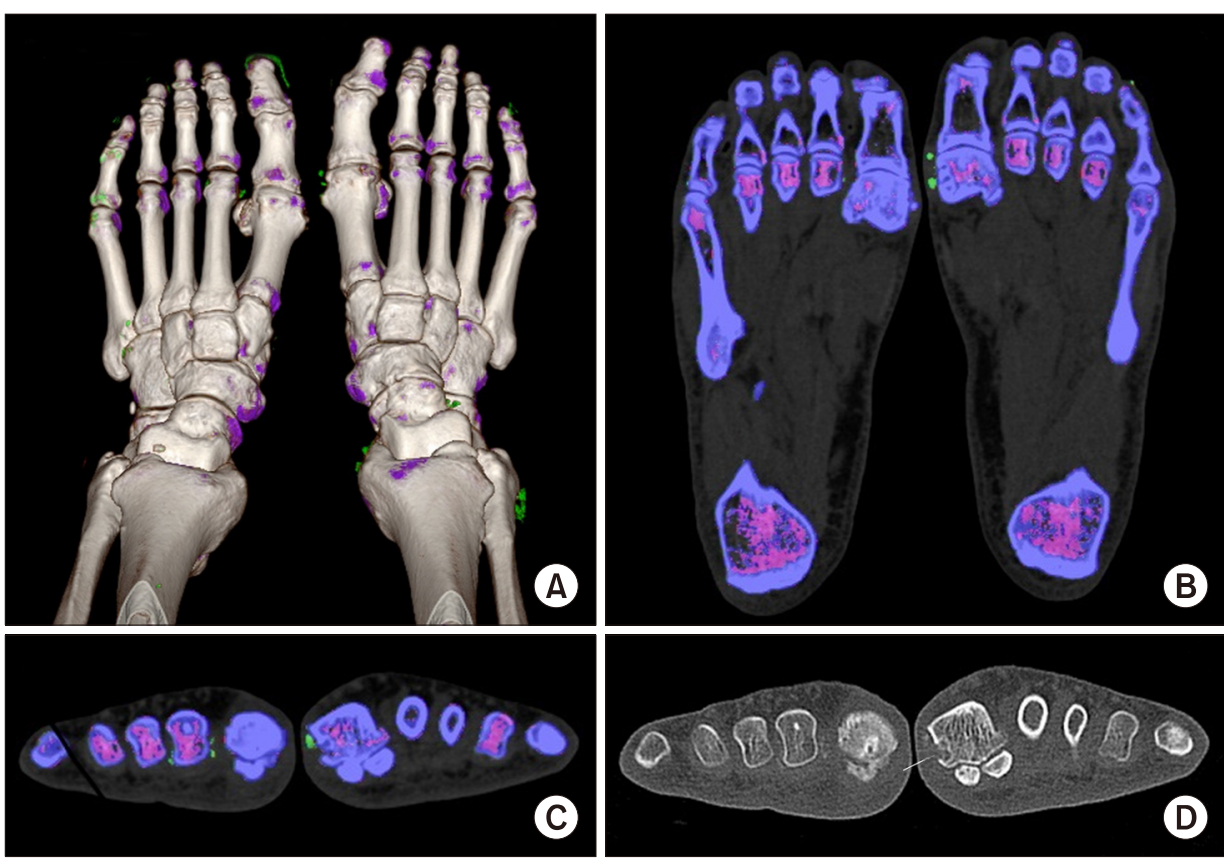

Figure 1. DECT of a 59-year old man with gout. Three-dimensional VRT image of both feet shows green MSU deposits adjacent right first MTP and left second MTP joints (A). Axial DECT image of both feet with small green microtophi (B). Coronal MPR DECT image presenting green microtophi around right first and left second MTP (C). Coronal MPR multi-detector CT image which shows erosion on right first MTP (arrow) (D). DECT: dual-energy computed tomography, VRT: volume rendering technique, $\mathrm{MSU}$ : monosodium urate, MTP: metatarsophalangeal, MPR: multiplanar reformation.

Table 3. Prevalence of erosions in joints with and without DECT MSU deposits $(n=51)$

\begin{tabular}{lccccc}
\hline \hline \multicolumn{1}{c}{ Site } & $\begin{array}{c}\text { With } \\
\text { MSU crystal }\end{array}$ & $\begin{array}{c}\text { Without } \\
\text { MSU crystal }\end{array}$ & Odds ratio & $\begin{array}{c}95 \% \\
\text { confidence interval }\end{array}$ & p-value \\
\hline MTP joints & $18 / 200(9.0)$ & $6 / 310(1.9)$ & 3.77 & $1.06 \sim 13.38$ & 0.040 \\
1st MTP & $18 / 53(34.0)$ & $6 / 49(12.2)$ & 3.77 & $1.06 \sim 13.38$ & 0.040 \\
Ankle joints & $8 / 56(14.3)$ & $6 / 46(13.0)$ & 1.06 & $0.37 \sim 3.04$ & 0.920 \\
Lisfranc joints & $2 / 21(15.7)$ & $14 / 81(17.3)$ & 0.50 & $0.10 \sim 2.40$ & 0.386 \\
Transverse tarsal joints* & $5 / 20(25.0)$ & $8 / 82(9.8)$ & 3.06 & $0.80 \sim 11.59$ & 0.100 \\
\hline
\end{tabular}

Values are presented as number/total (\%). Odds ratios and 95\% confidence interval were estimated by the generalized estimating equation model. DECT: dual-energy computed tomography, MSU: monosodium urate, MTP: metatarsophalangeal. *Intertarsal, talo-navicular, calcaneo-cuboidal. 
Table 4. Multivariate analysis for development of bone erosion in patients with DECT MSU deposits $(n=47)$

\begin{tabular}{|c|c|c|c|c|c|c|}
\hline \multirow[b]{2}{*}{ Variable } & \multicolumn{3}{|c|}{ Univariate } & \multicolumn{3}{|c|}{ Multivariate* } \\
\hline & Crude OR & $95 \% \mathrm{Cl}$ & p-value & $\begin{array}{l}\text { Adjusted } \\
\text { OR }\end{array}$ & $95 \% \mathrm{Cl}$ & $\mathrm{p}$-value \\
\hline Age & 1.10 & $1.03 \sim 1.17$ & 0.003 & 1.12 & $1.04 \sim 1.22$ & 0.004 \\
\hline Male & 0.00 & $0.00 \sim \infty$ & 0.999 & & & \\
\hline \multicolumn{7}{|l|}{ Comorbidity } \\
\hline Diabetes mellitus & 1.02 & $0.15 \sim 6.77$ & 0.984 & & & \\
\hline Hypertension & 5.04 & $1.40 \sim 18.14$ & 0.013 & & & \\
\hline Hyperlipidemia & 1.60 & $0.35 \sim 7.38$ & 0.547 & & & \\
\hline Previous CV & 4.91 & $0.54 \sim 44.60$ & 0.158 & & & \\
\hline Chronic kidney disease & 4.91 & $0.54 \sim 4.60$ & 0.158 & & & \\
\hline Duration of gout & 1.14 & $0.95 \sim 1.36$ & 0.170 & & & \\
\hline Frequency of gout attack & 1.84 & $0.93 \sim 3.66$ & 0.082 & 2.57 & $1.02 \sim 6.50$ & 0.047 \\
\hline Serum urate & 0.81 & $0.52 \sim 1.26$ & 0.354 & & & \\
\hline Average volume of MSU deposit $^{\dagger}$ & 4.05 & $0.29 \sim 56.05$ & 0.297 & & & \\
\hline \multicolumn{7}{|l|}{ Sites of MSU deposit } \\
\hline MTP & 0.46 & $0.04 \sim 4.82$ & 0.519 & & & \\
\hline Ankle & 0.41 & $0.11 \sim 1.57$ & 0.194 & & & \\
\hline Lisfranc & 0.46 & $0.13 \sim 1.60$ & 0.221 & & & \\
\hline Transverse tarsal joint $^{\ddagger}$ & 0.87 & $0.24 \sim 3.08$ & 0.825 & & & \\
\hline Achilles & 0.20 & $0.06 \sim 0.71$ & 0.013 & & & \\
\hline
\end{tabular}

DECT: dual-energy computed tomography, MSU: monosodium urate, OR: odds ratio, Cl: confidence interval, CV: cardiovascular, MTP: metatarsophalangeal. *The presented factors were selected through multivariate logistic regression analysis using backward selection process among the statistically significant factors with p-value $<0.1$ in univariate analysis. ${ }^{\dagger}$ Total volume of MSU deposit/number of MSU deposit, ${ }^{\ddagger}$ Intertarsal, talo-navicular, calcaneo-cuboidal.

Achilles tendon (OR 0.20, 95\% CI 0.06 0.71, p=0.013) correlated with presence of bone erosion in patients with MSU deposits. However, multivariate analysis showed that only older age and frequency of gout attack were associated with bone erosion in patients with MSU deposits (OR 1.12 and 2.57, 95\% CI 1.04 1.22 and 1.02 6.50, $\mathrm{p}=0.004$ and 0.047 , respectively). No significant association was detected between erosion and other clinical features of gout including duration of disease, serum urate level, and average urate volume in DECT.

\section{DISCUSSION}

This study has demonstrated that MSU deposits and erosions are frequently detected by DECT even in non-tophaceous symptomatic gout patients. Moreover, MSU deposits in 1st MTP joints were associated with presence of bone erosions, and older age and frequent gout attack increased the risk of development of bone erosion.

DECT allows sensitive detection of MSU deposits in patients with gout and hyperuricemia. In a DECT assessment of urate deposition in 20 patients with tophaceous gout, 4 times more loci of MSU deposits have been detected by DECT than by physical examination, especially in foot, ankle, and knee joints [13]. MSU deposition was also reported to be found in $25 \%$ of patients with asymptomatic hyperuricemia [9]. We were able to detect MSU depositions on DECT in $92 \%$ of gout patients evaluated even though these patients did not have clinically apparent tophi, supporting the findings of previous researches that DECT is a very sensitive method for detecting MSU deposits $[6,13]$. Furthermore, consistent with our results, patients who were treated with allopurinol and had no clinical tophi were revealed to have MSU crystal deposits on DECT scans in a recent study [11].

The prevalence of joint damage and its relationship with tophi in chronic tophaceous gout patients with a heavy burden of tophi is well defined [12,15,19-21]. In a DECT study of patients with tophaceous gout, erosions were found in $28.5 \%$ of total joints evaluated. MSU crystal depositions were more frequently detected in joint with radiographic change and the number of crystal deposition was correlated strongly with erosion score [12]. A CT study of chronic tophaceous gout patients revealed sim- 
ilar result showing $29.7 \%$ of erosion among total joints and strong relationship between intraosseous tophus and erosion [15]. However, this prevalence of bone erosion and its relationship with MSU deposits detected by DECT in patients with a low urate burden has not been investigated. In this study of patients with non-tophaceous symptomatic gout, over half of symptomatic gout patients without clinically apparent tophi had erosions, and erosions were found in $8.2 \%$ of their total joints evaluated, which was less prevalent than in patients with tophaceous gout. MSU deposits in the first MTPs, not in other joints were associated with the risk of bone erosion in symptomatic patients with non-tophaceous gout. The fist MTP joints are not only the most common site for symptom development and MSU deposition but also the common site of bone erosion in gout [22-24]. A systemic review and meta-analysis of the first MTP joint in gout revealed that the pooled prevalence of 1st MTP arthritis was $73 \%$, and that bone erosion in 1st MTPs ranged from $40 \%$ to $79 \%$ [22]. All erosions at 1st MTP were adjacent to tophaceous material in an ultrasonographic study [5], and the presence of erosion on X-ray in each joint was significantly correlated with urate volume in that joint among patients with tophaceous gout [25]. The significant association between MSU deposit and erosion only in 1st MTP might be derived from larger volume of MSU deposit in 1st MTP than in other joints considering the frequency and the predilection of MSU depositions in 1 st MTP.

In our analysis of risk factors for developing erosion in patients with MSU deposits on DECT among symptomatic gout patients without apparent tophi, older age and frequent gout attack were revealed as the independent risk factors. In a previous DECT study of gout patients treated with allopurinol, male sex, presence of palpable tophi, and the total volume of MSU crystal deposition were associated with higher prevalence of joint erosion [11]. Older age and higher number of clinical tophi were also positively related with erosion score in a radiographic study of gout patients including $50 \%$ of patients with tophi [26]. Dalbeth et al. [15] revealed a strong correlation between the number of $\mathrm{CT}$ erosions and C-reactive protein, which may reflect that the inflammatory response contributes to bone erosion, in paired radiographs and CT study of 20 patients with gout. It is also suggested by several in vivo studies that bone erosion in gout is mediated by activation of osteoclastogenesis and by compromised function of osteoblast releasing pro-in- flammatory cytokines [27]. We have found that in accordance with previous studies mainly in patients with chronic tophaceous gout, older age and frequent gout attack were associated with bone erosion in patients with non-tophaceous gout. In patients with lower urate burden as in our patient group, total volume of urate deposits may not contribute to the development of bone erosions.

This study has some limitations. First, the retrospective design of this study does not allow us to confirm the direction of relationship between MSU crystal depositions and structural damage showed in MTP joints. Second, as ankle and foot joints are prone to be involved in osteoarthritis, erosion might have been overestimated in such joints [28]. Finally, as the total erosion scoring was not used in this study, it was difficult to analyze the relationship between total number of MSU deposits and erosion score. Despite those limitations, this study has strength in that relationship between MSU deposits on DECT and bone erosions was evaluated in specific population of symptomatic non-tophaceous gout patients that we come across most often in the clinic.

\section{CONCLUSION}

In conclusion, MSU deposits and erosions were frequently detected by DECT in non-tophaceous symptomatic gout patients, and the presence of MSU deposits in 1st MTP joints was related with presence of bone erosions. Older age and frequent gout attack independently increased the risk of developing structural damage in patients with non-tophaceous symptomatic gout.

\section{CONFLICT OF INTEREST}

No potential conflict of interest relevant to this article was reported.

\section{REFERENCES}

1. Richette P, Bardin T. Gout. Lancet 2010;375:318-28.

2. Popp JD, Bidgood WD Jr, Edwards NL. Magnetic resonance imaging of tophaceous gout in the hands and wrists. Semin Arthritis Rheum 1996;25:282-9.

3. McQueen FM, Doyle A, Dalbeth N. Imaging in gout--what can we learn from MRI, CT, DECT and US? Arthritis Res Ther 2011;13:246.

4. Perez-Ruiz F, Martin I, Canteli B. Ultrasonographic measurement of tophi as an outcome measure for chronic gout. J Rheumatol 2007;34:1888-93. 
5. Thiele RG, Schlesinger N. Diagnosis of gout by ultrasound. Rheumatology (Oxford) 2007;46:1116-21.

6. Bongartz T, Glazebrook KN, Kavros SJ, Murthy NS, Merry SP, Franz WB 3rd, et al. Dual-energy CT for the diagnosis of gout: an accuracy and diagnostic yield study. Ann Rheum Dis 2015;74:1072-7.

7. Desai MA, Peterson JJ, Garner HW, Kransdorf MJ. Clinical utility of dual-energy CT for evaluation of tophaceous gout. Radiographics 2011;31:1365-75.

8. Breuer GS, Bogot N, Nesher G. Dual-energy computed tomography as a diagnostic tool for gout during intercritical periods. Int J Rheum Dis 2016;19:1337-41.

9. Dalbeth N, House ME, Aati O, Tan P, Franklin C, Horne A, et al. Urate crystal deposition in asymptomatic hyperuricaemia and symptomatic gout: a dual energy CT study. Ann Rheum Dis 2015;74:908-11.

10. Araujo EG, Bayat S, Petsch C, Englbrecht M, Faustini F, Kleyer A, et al. Tophus resolution with pegloticase: a prospective dual-energy CT study. RMD Open 2015;1: e000075.

11. Dalbeth N, Nicolaou S, Baumgartner S, Hu J, Fung M, Choi HK. Presence of monosodium urate crystal deposition by dual-energy CT in patients with gout treated with allopurinol. Ann Rheum Dis 2018;77:364-70.

12. Dalbeth N, Aati O, Kalluru R, Gamble GD, Horne A, Doyle AJ, et al. Relationship between structural joint damage and urate deposition in gout: a plain radiography and dual-energy CT study Ann Rheum Dis 2015;74:1030-6.

13. Choi HK, Al-Arfaj AM, Eftekhari A, Munk PL, Shojania K, Reid G, et al. Dual energy computed tomography in tophaceous gout. Ann Rheum Dis 2009;68:1609-12.

14. McQueen FM, Doyle A, Reeves Q, Gao A, Tsai A, Gamble $\mathrm{GD}$, et al. Bone erosions in patients with chronic gouty arthropathy are associated with tophi but not bone oedema or synovitis: new insights from a 3 T MRI study. Rheumatology (Oxford) 2014;53:95-103.

15. Dalbeth N, Clark B, Gregory K, Gamble G, Sheehan T, Doyle A, et al. Mechanisms of bone erosion in gout: a quantitative analysis using plain radiography and computed tomography. Ann Rheum Dis 2009;68:1290-5.

16. Wallace SL, Robinson H, Masi AT, Decker JL, McCarty DJ,
Yü TF. Preliminary criteria for the classification of the acute arthritis of primary gout. Arthritis Rheum 1977;20:895-900.

17. Inker LA, Astor BC, Fox CH, Isakova T, Lash JP, Peralta CA, et al. KDOQI US commentary on the 2012 KDIGO clinical practice guideline for the evaluation and management of CKD. Am J Kidney Dis 2014;63:713-35.

18. Mallinson PI, Coupal T, Reisinger C, Chou H, Munk PL, Nicolaou S, et al. Artifacts in dual-energy CT gout protocol: a review of 50 suspected cases with an artifact identification guide. AJR Am J Roentgenol 2014;203:W103-9.

19. Levin MH, Lichtenstein L, Scott HW. Pathologic changes in gout; survey of eleven necropsied cases. Am J Pathol 1956;32:871-95.

20. Sokoloff L. The pathology of gout. Metabolism 1957;6:230-43.

21. McQueen FM, Chhana A, Dalbeth N. Mechanisms of joint damage in gout: evidence from cellular and imaging studies. Nat Rev Rheumatol 2012;8:173-81.

22. Stewart S, Dalbeth N, Vandal AC, Rome K. The first metatarsophalangeal joint in gout: a systematic review and meta-analysis. BMC Musculoskelet Disord 2016;17:69.

23. Dalbeth N, Merriman TR, Stamp LK. Gout. Lancet 2016; 388:2039-52.

24. Kim SK, Lee H, Kim JH, Park SH, Lee SK, Choe JY. Potential interest of dual-energy computed tomography in gout: focus on anatomical distribution and clinical association. Rheumatology (Oxford) 2013;52:402-3.

25. Sapsford M, Gamble GD, Aati O, Knight J, Horne A, Doyle AJ, et al. Relationship of bone erosion with the urate and soft tissue components of the tophus in gout: a dual energy computed tomography study. Rheumatology (Oxford) 2017;56:129-33.

26. Spaetgens B, van Durme C, Webers C, Tran-Duy A, Schoonbrood T, Boonen A. Construct validity of radiographs of the feet to assess joint damage in patients with gout. J Rheumatol 2017;44:91-4.

27. Ragab G, Elshahaly M, Bardin T. Gout: an old disease in new perspective - a review. J Adv Res 2017;8:495-511.

28. Roddy E, Zhang W, Doherty M. Are joints affected by gout also affected by osteoarthritis? Ann Rheum Dis 2007;66: 1374-7. 